\title{
Momentum-dependent potentials: Towards the molecular dynamics of fermionlike classical particles
}

\author{
P. Cordero \\ Departamento de Física, Facultad de Ciencias Físicas y Matemáticas, Universidad de Chile, \\ Casilla 487, Santiago 3, Chile \\ E. S. Hernández \\ Departamento de Física, Facultad de Ciencias Exactas y Naturales, Universidad de Buenos Aires, \\ 1428 Buenos Aires, Argentina
}

(Received 5 July 1994)

\begin{abstract}
We investigate classical Hamiltonian models for particles interacting with steep differential repulsive barriers both in coordinate and momentum space. The final aim is to define a classical system of many particles behaving as fermions in many respects. In this paper we examine the appearance of the phase portrait of one- or two-particle systems to skim the essential features that would later be transcribed to the basic rules of a molecular dynamics algorithm. One of the remarkable properties of the phase portrait is the flow from states that start far away with a wide range of momentum towards a narrow region in momentum - a virtual locking of momentum - in the vicinity of the steepest part of the barrier in momentum space. The central ideas are developed through two examples in one and two dimensions.
\end{abstract}

PACS number(s): 03.65.Sq, 05.30.Fk, 05.90.+m

\section{INTRODUCTION}

The ancient distinction between theoretical and experimental physics traditionally furnished the unique scenario to locate the objects of research in this science. This frame is no longer unique in view of the increasing importance awarded to computational physics. In particular, experiments on molecular dynamics or Monte Carlo simulations of many-particle systems considerably enlarge the field of many-body physics; lying halfway between laboratory experiments and analytic theories, numerical experiments are capable of describing both qualitative and quantitatively the phenomenology of a variety of large physical objects, making room for comprehension of the detailed microscopic events responsible for observable effects.

Since computational facilities may severely constrain the possibilities to invest efforts in numerical experiments for systems with many degrees of freedom, simplified models involving, for example, two-dimensional fluids together with a discrete dynamical map deserve special recognition. In particular, the dynamics of particles subject to piecewise constant potentials - bare hard disks in the simplest version-can be dealt with numerically by means of a nondifferential, event driven algorithm that allows an excellent computational performance [1]. The phenomenology covered by $N$ hard disk systems ranges from hydrodynamic instabilities to nucleation phenomena, through transport processes and dynamical response [2]. In spite of the considerable power of this approach, systems of indistinguishable particles described by fully symmetrized or antisymmetrized state vectors are not amenable, in principle, to a molecular dynamics experiment [3].

Attempts to submit an $N$-fermion system to a dynam- ical simulation have been put forward by nuclear physicists aiming at descriptions of the evolution of excited and compressed nuclear matter. On the one hand, the fermionic molecular dynamics developed by Feldmeier [4] is a semiclassical map of the actual $N$-fermion motion on the manifold of Slater determinants of single-particle wave packets and includes the antisymmetrization effects both in the exchange matrix elements of the interaction and in the matrix of the symplectic metrics. It gives an interesting qualitative description of many phenomena occurring in nuclear reactions [5]; however, since the method relies on a mean field variational approach, it may be subject to the short-time regime as indicated by several authors (see, for example, [6] and references cited therein). On the other hand, the quantum molecular dynamics $[3,8,9]$ together with the Vlasov-UehlingUhlenbeck or Boltzmann-Uehling-Uhlenbeck $[10,11]$ approaches represent serious advances in this direction; the fermions are regarded as classical particles undergoing Hamiltonian motion in an effective, i.e., densitydependent, mean field and may participate in two-body collision events controlled by typical scattering cross sections, together with a criterion to rule out those processes forbidden by Pauli exclusion. Another useful viewpoint [12-15] intends to mimic the antisymmetric character of the many-fermion wave function allowing the particles to repel each other according to a potential that depends on the relative momentum of the interacting pair. The momentum dependent potential may be parametrized so as to account for the equation of state of nuclear matter at zero temperature predicted by variational Monte Carlo methods [16]. It is worthwhile recalling that the first investigation of high energy nuclear dynamics, i.e., above 300-500 MeV per particle, was carried by Bodmer et al. [7]. In this work, a momentum dependent interac- 
tion was considered, however, as an artifact to reproduce the asymptotic two-body trajectory or deflection function since exclusion effects are not relevant at the given energies.

Although the momentum potential models have proven to work satisfactorily to describe many interesting phenomena in nuclear dynamics, such as fragmentation of hot compressed nuclear matter [17], several questions may be raised concerning the validation of this philosophy. To the best of our knowledge, the Hamiltonian dynamics induced by these potential models has not been investigated beyond the examples of phase space trajectories presented in Refs. $[12,14]$. It is consequently interesting to examine them, referring to simple dynamical systems in order to ascertain whether they account for exclusion effects. It seems appropriate then to examine the basic features of the dynamics of systems of particles that repel each other in momentum space according to a simple momentum-dependent potential. The most relevant aspects of such dynamics may then be translated into simple collision rules to be inserted into an event driven algorithm such as the one in Ref. [1]. A simple justification for the use of these potentials may rely on the fact that in a real quantum liquid, exchange correlations manifest themselves in the total energy by means of nonlocal matrix elements of the pair interaction; this nonlocality in turn gives rise to inertial effects, namely, to renormalization of the bare mass into an effective, momentum dependent, mass. These effects can be taken care of by momentum-dependent interaction terms. This view could give support to the choice of momentum dependent potentials to face an approach to the dynamics of a quantum liquid with classical particles.

The purpose of this work is to advance the understanding of the virtues and capabilities of momentum dependent potentials to provide some of the features of fermion repulsion. The final goal is the formulation of collision rules for molecular dynamics simulations of hard particle fluids, aiming at future descriptions of quantum liquids. This task will be the subject of a forthcoming paper.

The present article will be mostly concerned with momentum dependent fields that prevent particles from reaching a given set of momentum values; we then investigate a selection of continuous potential models such that in each of them, the hard disk repulsion is softened and represented by a barrier with smooth edges. For the sake of completeness, in Sec. II we discuss a one-dimensional model with quadratic coordinate and momentum potentials in a finite region of phase space, which can be tackled analytically. In Sec. III a one-dimensional model for soft disk repulsion together with a smooth momentum barrier or well is presented and discussed. One- and two-particle systems in two dimensions, resembling closely the hard disk problem, are examined in Sec. IV and a full discussion is the subject of Sec. V.

\section{EXACTLY SOLVABLE ONE-DIMENSIONAL MODEL}

In this section we present and discuss a simple onedimensional model for a particle moving in the presence of two short range potentials in both coordinate and momentum space. These potentials are of the form

$$
V_{y}(y)=\left\{\begin{array}{l}
\frac{E_{y}}{2}\left(1-y^{2}\right) \text { if } \quad|y|<1 \\
0 \quad \text { otherwise }
\end{array}\right.
$$

for $y=x$ or $p$. The two-dimensional phase space then splits into four different regions, namely, (i) the four force-free domains $|x|>1$ and $|p|>1$; (ii) the vertical strips $|x|<1$, and $|p|>1$ where only the accelerating force is present; (iii) the horizontal strips $|x|>1$, and $|p|<1$ where the momentum field is nonvanishing; and (iv) the unit square where both potentials are active. The specific orbits in regions (i)-(iv) are described in what follows.

Region (i). The phase flow consists of horizontal straight lines corresponding to uniform motion with velocity $\dot{x}=p$.

Region (ii). The particle Hamiltonian is

$$
H=\frac{p^{2}}{2}+\frac{E_{x}}{2}\left(1-x^{2}\right)
$$

Accordingly, the phase trajectories are either hyperbolas for the repulsive barrier or ellipses for the attractive well. In the former situation, two separatrices show up at $p=$ $\pm \sqrt{E_{x}}$, corresponding to an energy $E=E_{x}$.

Region (iii). With

$$
H=\frac{p^{2}}{2}+\frac{E_{p}}{2}\left(1-p^{2}\right)
$$

the orbits are straight lines. The only effect of the momentum dependent potential is to renormalize the particle mass according to the velocity equation

$$
\dot{x}=p\left(1-E_{p}\right) .
$$

It is then clear that for a repulsive barrier, the effective mass changes sign if $E_{p}>1$, causing the velocity to lie antiparallel to the momentum.

Region (iv). The Hamiltonian can be written as

$$
H=\frac{E_{x}+E_{p}}{2}+\frac{\left(1-E_{p}\right)}{2} p^{2}-E_{x} x^{2} .
$$

In this case the orbits present several possibilities according to the sign of $E-\left(E_{x}+E_{p}\right) / 2$ for each energy $E$ and to the signs of $E_{x}, E_{p}$, their shapes being either hyperbolas or ellipses.

In this model, the full features of the phase portrait for each choice of signs and magnitudes of the potential parameters can be explicitly worked out. In particular, fixed points and separatrices can be located; it is easy to realize that the Hamiltonian flow is structurally unstable since the character of the $(0,0)$ fixed point changes according to the parameter set, from a saddle to a stable elliptic point, the latter appearing when $E_{x}\left(1-E_{p}\right)$ is positive. In Fig. 1 we illustrate the phase diagrams for the four possible sign combinations and indicate the characteristic lines. The appearance of the orbits within the unit square depends also on the relative sizes of the strengths $E_{x}$ and $E_{p}$. 
The most interesting outcome of this study is the existence of librations even in the case of purely repulsive barriers both in coordinate and in momentum space as shown in Fig. 1. It is worth noting that bound periodic motion takes place for positive $E_{p}$ larger than unity; since the effective particle mass is negative under such conditions, the velocity antiparallel to the momentum prevents the particle from escaping downhill the two-dimensional barrier on the energy surface. This effect is intrinsic to energy surfaces with other than purely kinetic, i.e., $p^{2}$ momentum dependence, and has been thoroughly illustrated for systems with spectrum generating algebras of the $\mathrm{su}(2)$ or $\mathrm{su}(1,1)$ type (see, for example, Refs. [18-20] and references therein).

\section{SMOOTHED SQUARE POTENTIALS}

In the following we discuss the phase portraits for a kind of particle Hamiltonians involving soft sphere repulsion and a momentum dependent field. These potentials are selected in such a way that their limiting form is a square well or barrier. The analytic form chosen for the current investigation is

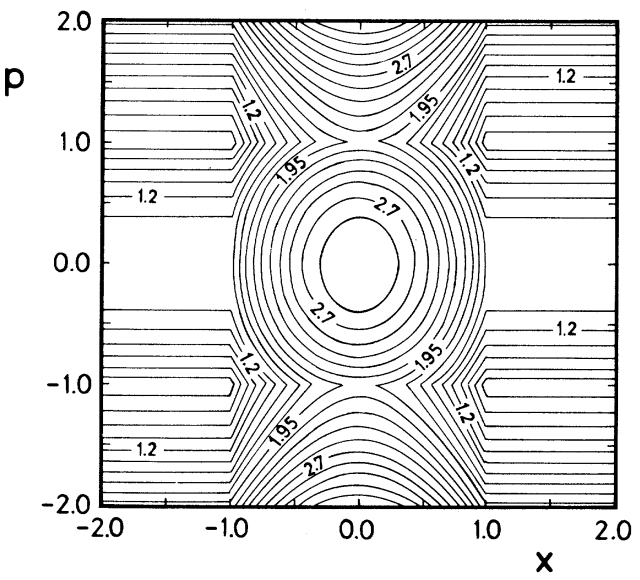

(a)

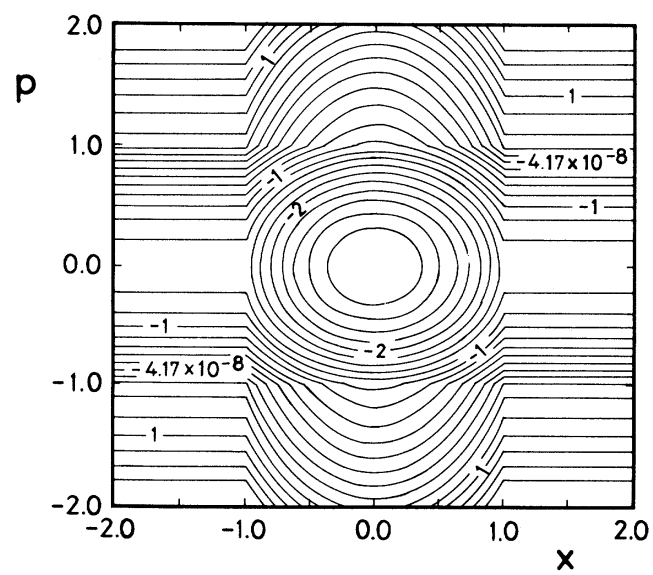

$$
V_{y}(y)=\frac{E_{y}}{1+e^{b_{y}\left(y^{2}-1\right)}}
$$

for $y=x, p$. Infinitely large values of either parameter $b_{y}$ give rise to a perfect square barrier or well, whose strength can also be adjusted controlling the corresponding intensity $E_{y}$. The particle Hamiltonian is then

$$
H=\frac{p^{2}}{2}+V_{x}(x)+V_{p}(p)
$$

everywhere. We shall consider only positive $E_{x}$ and both signs for $E_{p}$.

Typical energy surfaces and contour plots are shown in Figs. 2-4. In Fig. 2 (a) we display the energy surface corresponding to equal strengths $E_{x}=E_{p}=3$ (repulsive momentum barrier) and core widths $b_{x}=b_{p}=10$. The nature of the fixed points can be better appreciated in Fig. 2(b), where the phase portrait is plotted; it can be noticed that the flow here shown is equivalent to that in Fig. 1(b), while different choices of the model parameters give rise to contour plots equivalent to those in Figs. 1(a), $\mathbf{1}(\mathrm{c})$ or $1(\mathrm{~d})$. In the present case, we realize that five fixed points appear along the $p$ axis, namely, a saddle point at the origin of phase space at an energy equal to the sum

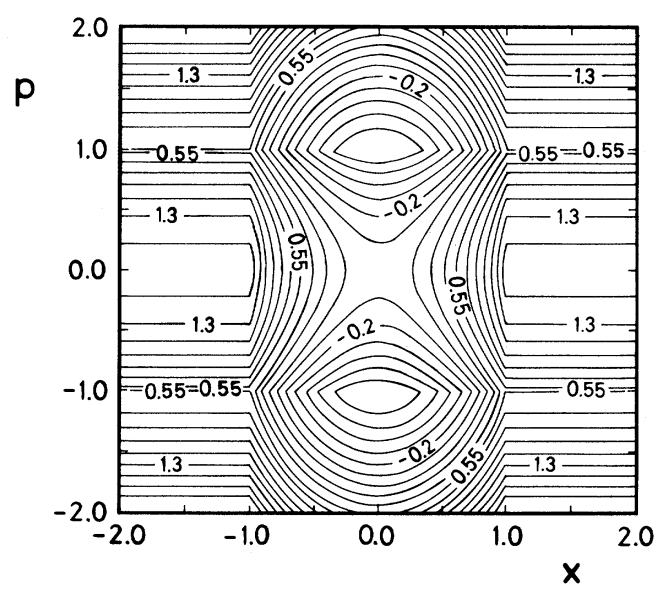

(b)

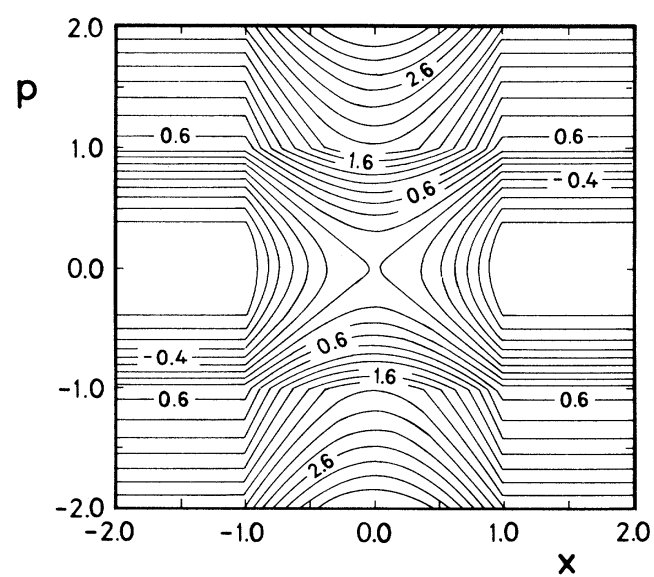

(d)

FIG. 1. Phase diagrams for the quadratic Hamiltonian in Sec. II (see the text for details). All variables and contour labels are dimensionless. (a) $E_{x}=E_{p}=3$; (b) $E_{x}=-E_{p}=-3$; (c) $E_{x}=E_{p}=-3$; (d) $-E_{x}=E_{p}=3$. 


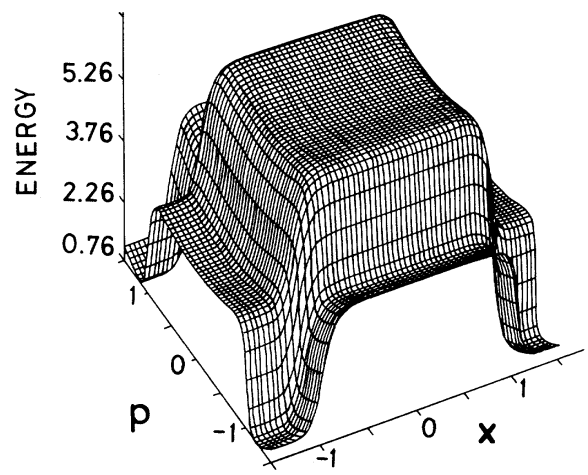

(a)

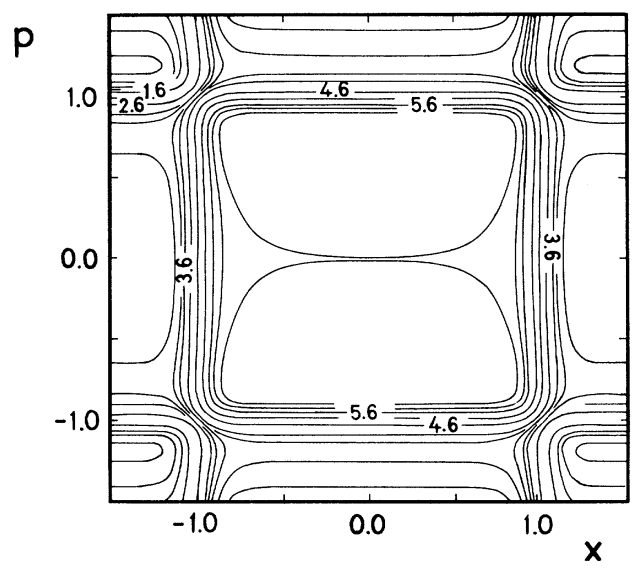

(b)

FIG. 2. Smoothed square potential Hamiltonian given in Eq. (3.2) for $E_{x}=E_{p}=3, b_{x}=b_{p}=10$. (a) Energy surface (arbitrary units as $E_{x}$ and $E_{p}$ ); (b) phase diagram.

of both barrier heights, two hyperbolic points symmetrically located at a value of $|p|$ sligthly above unity, and two symmetric stable elliptic points within the unit square. Notice as well that the phase trajectory through the origin is a separatrix corresponding to the borders of the basins of attraction of the symmetric stable points. The constant $p$ lines across these fixed points are zero velocity lines; their precise locations are given by the solutions of the equation

$$
\begin{aligned}
\dot{x} & =p\left(1-\frac{2 E_{p} e^{b_{p}\left(p^{2}-1\right)}}{\left[1+e^{b_{p}\left(p^{2}-1\right)}\right]^{2}}\right) \\
& =0
\end{aligned}
$$

As one solves Eq. (3.3), one soon realizes that the values of the solutions are ruled by the product $E_{p} b_{p}$. Insofar as this product is larger than 2 , the energy surface and phase portrait are similar to the ones in Fig. 2. If $E_{p} b_{p}=2$, the hyperbolic points outside the square radius collapse on $p= \pm 1$, while for smaller values of this product, no real fixed points exist other than the origin. This is illustrated in Fig. 3, where for $E_{x}=E_{p}=0.2, b_{x}=b_{p}=10$ we observe that the closed orbits and the separatrix through $p= \pm 1$ have already disappeared. The role of the phase trajectory through the origin now is to separate the pure scattering trajectories

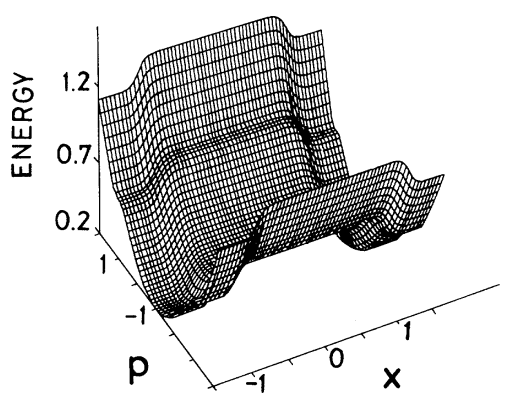

(a)

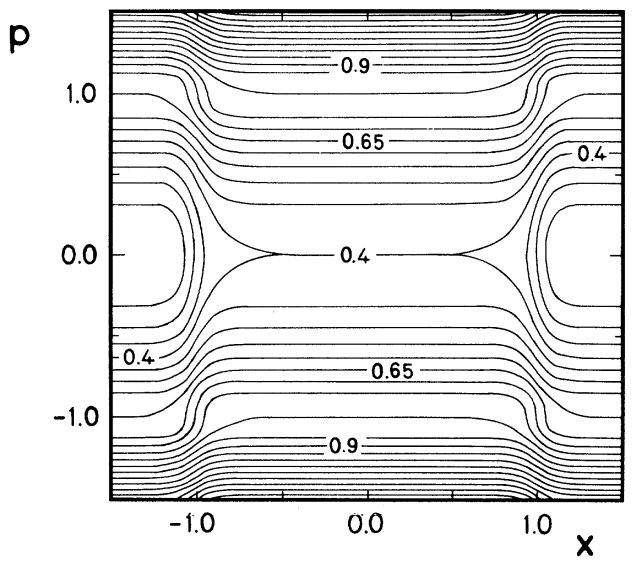

(b)

FIG. 3. Same as in Fig. 2, but with $E_{x}=E_{p}=0.2$.

where the particle bounces at the coordinate potential barrier from those where the incoming energy is enough to overcome the repulsion and allow crossing to the negative $x$ region. In addition, it is easy to verify that the fixed point at $p=0$ changes character from hyperbolic to elliptic as $E_{p} b_{p}$ increases above the value $1+\cosh \left(b_{p}\right)$; it is convenient to keep in mind that very intense fields (large $E_{p}$ ) together with hard walls (large $b_{p}$ ) constitute our desirable scenario for future dynamical simulations of $N$-particle systems.

In Fig. 4 we show the same surfaces and contour lines as in Fig. 2, but for a momentum well with $E_{p}=-3$. The phase portrait is quite similar to that in Fig. 3; indeed, in this case one can analytically show that the origin of phase space is the only fixed (hyperbolic) point for this dynamics.

We end this section presenting an example that may bear some relevance to classical models of fermi-like particles, as it will be discussed in further detail in Sec. IV. The model corresponds to a localized momentum potential well of the form

$$
V_{p}(p)=\frac{E_{p}}{1+e^{b_{p}\left[\left(p-p_{0}\right)^{2}-1\right]}},
$$

and a typical phase diagram is displayed in Fig. 5. Apart from the symmetry break induced by the shift in the variable of the momentum dependent potential, we can appreciate that the negative $p$ region of this diagram preserves most of the characteristics of the case $p_{0}=0$ shown in Fig. 4, namely, the presence of a separatrix through 

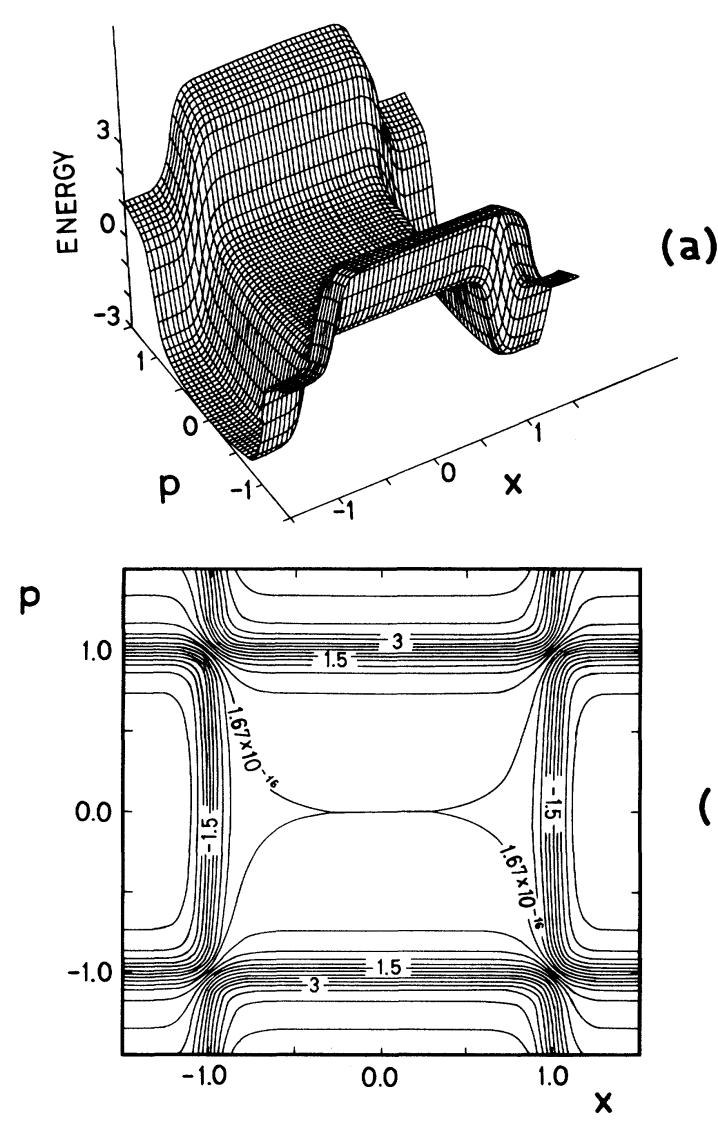

(b)

FIG. 4. Same as Fig. 2, but with $E_{x}=-E_{p}=3$.

the origin, an elliptic fixed point between $p=0$ and $p=-1$, and a saddle node for $p<-1$. In this case, the critical points are given by the equation

$$
p=p_{0} \frac{2 E_{p} b_{p} \tilde{V}(1-\tilde{V})}{2 E_{p} b_{p} \tilde{V}(1-\tilde{V})-1}
$$

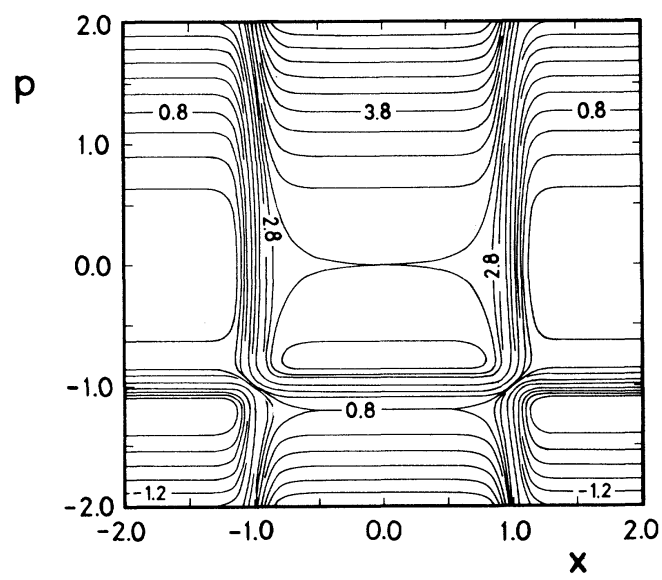

FIG. 5. Phase diagram for a Hamiltonian with a smoothed potential barrier plus the localized momentum dependent potential given in Eq. (3.4) for $E_{x}=-E_{p}=3, b_{x}=b_{p}=10$, and $p_{0}=-2$. with $V_{p}(p)=E_{p} \tilde{V}$. Equation (3.5) may be rewritten in terms of the displaced variable $u^{2}=\left(p-p_{0}\right)^{2}$ as

$$
u^{2}=\frac{p_{0}^{2}}{\left[2 E_{p} b_{p} V\left(\tilde{u}^{2}\right)\left(1-V\left(\tilde{u}^{2}\right)\right)-1\right]^{2}} .
$$

The solutions of the zero velocity equation can be obtained numerically; however, a close examination shows that if

$$
E_{p} b_{p}-2= \pm 2 p_{0}
$$

a saddle node bifurcaton takes place with an unstable point $u^{2}=1$. For $\left|E_{p} b_{p}-2\right|>2 p_{0}$, the latter bifurcates into a stable point $u^{2}<1$ and an unstable one $u^{2}>1$. On the other hand, we can observe in Eq. (3.6) that $u^{2} \approx p_{0}^{2}$, i.e., $p \approx 0$, unless the denominator, which is precisely the bracket in Eq. (3.3), vanishes. For the displaced momentum well, the bifurcation set (3.7) generalizes the condition $E_{p} b_{p}=2$.

An important feature of this pattern is the fact that the scattering orbits $|x|>1$, and $p \leq-1$ accumulate in the vicinity of the edge $p=-1$ of the momentum well. The related dynamical picture for a particle shot towards the barrier with an initial negative momentum inside the well corresponds to a final momentum locked on the hillside. As a consequence, when the particle lies sufficiently far from the barrier on its way back, its kinetic energy is lower than the initial one. This dynamics is also encountered in the strips $|p|>1$ and $|x|>1$ of the phase diagram in Fig. 2(b).

\section{HIGHER-DIMENSIONAL MODELS}

We now examine two particular models devised to understand the competition between a smooth potential barrier in coordinate space and the effective mass effects induced by the momentum dependent field for (a) one particle moving in a plane and (b) two particles mutually repelling on a plane, each of them immersed in a localized momentum dependent well. The former example can be regarded as a model for a particle outside the Fermi sphere of an $N$-fermion system (momentum barrier) or as a model for a particle blocked inside the Fermi sea, its momentum thus confined to the cell allowed by the boundary conditions and the macroscopic, i.e., density and temperature, regime (momentum well). The second example corresponds rather to a blocked two fermion system, as discussed below.

\section{A. Particle on a plane}

Let us consider a Hamiltonian of the form (3.2) in two dimensions. The particle can now approach the scattering center at the origin with a nonvanishing impact parameter. In the absence of mass renormalization, the trajectories in coordinate space would resemble the reflection by a hard disk whenever the impact parameter is smaller than unity, the amount of curvature at the bounce depending upon the size of the smoothing pa- 
rameter $b_{x}$. In Fig. 6 we illustrate this situation for the choice $E_{x}=E_{p}=3, b_{x}=b_{p}=10$ (a) in coordinate space and (b) in momentum space. The initial position and momentum have been fixed at $x_{0}=2, p_{x 0}=-2, p_{y 0}=0$. The outstanding feature in Fig. 6(a) is the significant deviation of the low impact parameter trajectories with respect to the reflection expected for a hard disk scattering center. Indeed, for $y<0.5$ the particle acquires a negative $y$ velocity. Even for an impact parameter equal to unity, the particle is deflected forward, revealing the existence of a nonvanishing repulsion within the finite surface width of the potential barrier. The trajectories are no longer deviated for impact parameters larger than 1.1 .

This analysis can be complemented regarding Fig. 6(b). In the following we show that for the lowest impact parameters, the two-dimensional momentum becomes frozen at the surface of the momentum dependent barrier. This behavior is consistent with the feature encountered in the one-dimensional model discussed in Sec. III [cf. Figs. 2(b) and 5], namely, the fact that scattering trajectories impinging on the barrier lock the outcoming momentum at the edge of the momentum dependent field. A similar morphology is apparent for negative $E_{p}$ 's and will be discussed in the next subsection in the context of a localized momentum well.

\section{B. Two interacting particles on a plane}

We now consider a two-particle system with a smooth barrier interaction of the form

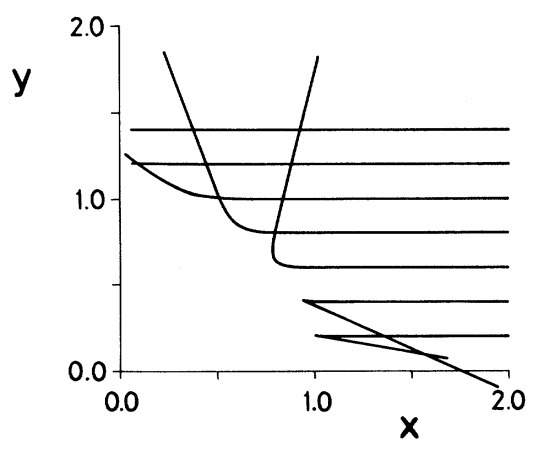

(a)

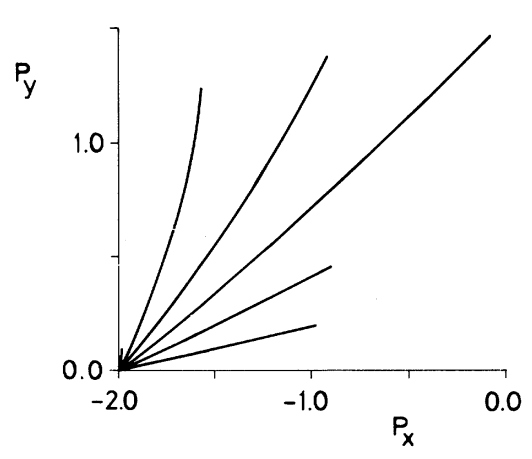

(b)

FIG. 6. Two-dimensional trajectories (a) in coordinate space and (b) in momentum space, for a two-dimensional particle subject to the Hamiltonian (3.2).

$$
V_{x}\left(\vec{r}_{1}, \vec{r}_{2}\right)=\frac{E_{x}}{1+e^{b_{x}\left[\left(x_{1}-x_{2}\right)^{2}+\left(y_{1}-y_{2}\right)^{2}-1\right]}} .
$$

Furthermore, we choose the Hamiltonian

$$
H=\frac{\vec{p}_{1}^{2}+\vec{p}_{2}^{2}}{2}+V_{x}\left(\vec{r}_{1}, \vec{r}_{2}\right)+V_{p}\left(\vec{p}_{1}\right)+V_{p}\left(\vec{p}_{2}\right),
$$

with $V_{p}(\vec{p})$ given by

$$
V_{p}(\vec{p})=\frac{E_{p}}{1+e^{b_{p}\left[\left(p_{x}-p_{x 0}\right)^{2}+p_{y}^{2}-1\right]}} .
$$

This Hamiltonian has been devised to mimic two particles immersed in the Fermi sphere characterizing a manyfermion system at low temperature; each particle momentum is thus allowed to vary within a narrow cell in momentum space. The well (4.3) simulates this situation for cells of average diameter equal to unity with a small border width $b_{p}$. The magnitude of this width, rather than the size of the cell, takes into account the relative washing out of Pauli blocking due to an increase in temperature. For the sake of an illustration, we consider a geometrically symmetric configuration where the particles are initially located with opposite position and momentum vectors. The eight-dimensional system of Hamilton's equations of motion reduces then to the four-dimensional one for a single particle. The problem then resembles that depicted in Fig. 6-a particle hitting a repulsive barrier-however, with the effective mass characteristics of the example in Fig. 5. We show some spatial oneparticle trajectories in Fig. 7, for the parameter choice $E_{p}=-3, p_{x 0}=-2$, and $p_{y 0}=0$, for the particle under consideration, the remaining parameters remaining the same as in Fig. 6. We now see that the effect of the repulsive barrier at the origin is preserved to the same extent; however, the bounce at the barrier surface does not correspond to a perfect reflection. One also verifies that after the particle has scattered its momentum saturates with modulus $|p| \approx 1$, consistent with the phase trajectories indicated in Fig. 5.

An interesting feature of the flow in these models is the fact that for some initial momentum values, incoming particles may bounce twice on the soft disk barrier. An example of this situation is shown in Fig. 8(a) [Fig. 8(b)]

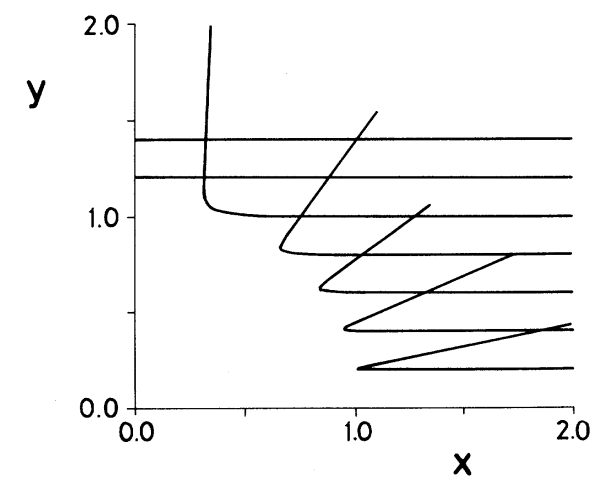

FIG. 7. Same as Fig. 6(a), but for two particles subject to the Hamiltonian in Eq. (4.2). The particles are assumed to be symmetrically located in both space and momentum space at $t=0$. 


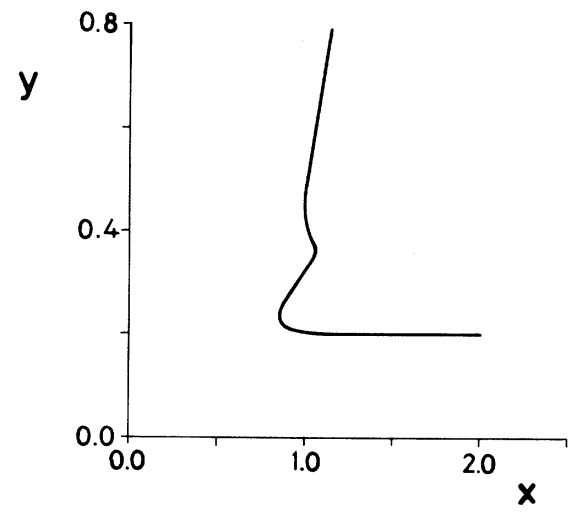

FIG. 8. Two-dimensional trajectory of one particle for the orbit with initial momentum $(-2.7,0)$ under the conditions of Fig. 7.

for the $(x, y)\left[\left(p_{x}, p_{y}\right)\right]$ projection of a phase space orbit with initial momentum $(-2.7,0)$ for a two-dimensional particle with the potential parameters of Figs. 6 and 7. This phenomenon has been observed with other versions of momentum dependent potentials, more specifically, for two different choices with the relative momentum as the variable $[12,14]$. Doubly bouncing orbits such as the one depicted in Fig. 8 are the two-dimensional versions of phase trajectories through two zero velocity lines, such as those appearing in Figs. 2(b) and 5 in the neighborhood of $( \pm 1,-1)$ and in the region of localized phase orbits with $|x|<1$.

\section{DISCUSSION}

As stated in the Introduction, the current motivation to tackle a study of momentum dependent potentials is to establish the extent to which they are able to simulate the quantum behavior of indistinguishable particles. The majority of the potentials proposed here serve this purpose for fermionlike particles: while barriers inhibit a finite momentum domain, wells explicitly allow a finite momentum region excluding the remaining space. The surface width of the barriers or wells is a control parameter whose vanishing gives rise to a steep potential step.

One of the predominant characteristics of Hamiltonian flows of one-dimensional systems is the peculiar role played by the inflection point of the momentum field (chosen as unity in the displayed examples). In fact, one can see that the density of horizontal flow lines is high in the neighbourhood of these points. As already mentioned in Sec. III, this means that phase trajectories starting in a field-free region but pointing towards the repulsive, soft disk barrier accumulate near the abovementioned inflection point after the bounce. The price to be paid on dynamical grounds is a lowering of the kinetic energy, which could be attributed to an increase in the effective mass that took place near the scattering center. A similar effect is encountered in the two-dimensional systems discussed in Sec. IV upon examination of the trajectories in either configuration or momentum space. It is also worthwhile mentioning that the Gaussian momentum fields employed in nuclear dynamics calculations [14] possess this feature as well.

This picture suggests that, for example, a projectile hitting a Fermi sphere with whose particles it interacts, on the average, through a soft repulsion, becomes captured on the surface of the sphere. On the other hand, if a particle is blocked inside the Fermi sea within a narrow allowed momentum cell, whose edges furnish a nonvanishing contribution to the particle velocity via the derivative of a smoothed stepwise momentum field, soft disk scattering events tend to lock the particle momentum on the boundary of its cell.

In spite of its simplicity, we believe that the models presented in this article encourage further research along these lines, destined to carry, for example, classical molecular dynamical experiments in two dimensions and to compare the bulk properties and both the thermal and the dynamical responses of the many body system obtained by these means with the predictions of some quantum many body theory - e.g., a mean field theorywhere antisymmetrization effects have been fully taken care of. This work is presently in progress and the corresponding results will be presented elsewhere.

\section{ACKNOWLEDGMENTS}

This work was partially supported by FONDECYT, Chile, through Project No. 1931105, and by CONICET, Argentina, through Grant No. PID 1993. One of us (E. S. H.) is pleased to acknowledge the Comisión Nacional de Investigaciones Científicas y Técnicas (CONICYT), Chile and the University of Chile for financial support and hospitality while this work was being done.
[1] M. Marín, D. Risso, and P. Cordero, J. Comput. Phys. 109, 306 (1993).

[2] D. Risso, Ph.D. dissertation, Universidad de Chile, 1994; D. Risso and P. Cordero, in Instabilities and Nonequilibrium Structures IV, edited by E. Tirapegui and W. Zeller (Kluwer Academic, Dordrecht, 1993); in Condensed Matter Theories, edited by A.N. Proto and J. Aliaga (Plenum, New York, 1991).

[3] J. Aichelin, C. Hartnack, A. Bohnet, L. Zhixia, G. Peilert, and H. Stocker, Phys. Lett. B 224, 34 (1989).
[4] H. Feldmeier, Nucl. Phys. A 515, 147 (1990).

[5] H. Feldmeier and J. Schnack, in Dynamical Features of Nuclei and Finite Fermi Systems, edited by X. Viñas, M. $\mathrm{Pi}$, and A. Ramos (World Scientific, Singapore, 1994).

[6] H. G. Solari and E. S. Hernández, Phys. Rev. C 26, 2310 (1982).

[7] A. R. Bodmer, C. N. Panos, and A. D. MacKellar, Phys. Rev. C 22, 1025 (1980).

[8] J. Aichelin and H. Stocker, Phys. Lett. B 176, 14 (1986).

[9] J. Aichelin, Phys. Rep. 202, 233 (1991). 
[10] H. Kruse, B. V. Jacak, and H. Stocker, Phys. Rev. Lett. 54, 289 (1985).

[11] C. Gregoire, B. Remaud, F. Sebille, L. Vinet, and Y. Raffray, Nucl. Phys. A 468, 321 (1987).

[12] L. Wilets, E. M. Henley, M. Kraft, and A. D. Mackellar, Nucl. Phys. A 282, 350 (1977).

[13] L. Wilets, Y. Yariv, and R. Chestnut, Nucl. Phys. A 301, 359 (1978).

[14] C. O. Dorso, S. Duarte, and J. Randrup, Phys. Lett. B 188, 287 (1987).

[15] C. Dorso and J. Randrup, Phys. Lett. B 215, 611 (1988).
[16] B. Friedman and V. R. Pandharipande, Nucl. Phys. A 361, 502 (1981).

[17] C. O. Dorso and J. Randrup, Phys. Lett. B 232, 29 (1989); 301, 328 (1993).

[18] H. S. Solari and E. S. Hernández, Phys. Rev. C 28, 2472 (1983).

[19] D. M. Jezek, E. S. Hernández, and H. G. Solari, Phys. Rev. C 34, 297 (1986).

[20] D. M. Jezek and E. S. Hernández, Phys. Rev. A 42, 96 (1990). 